\title{
Genel Hizalama İndeksi Yöntemiyle 2 Boyutlu Saf Fermiyonik Modelde Kaosun İncelenmesi
}

\author{
Beyrul Canbaz ${ }^{1 *}$ \\ 1* İstanbul Yeni Yüzyıl Üniversitesi, Sağlık Bilimleri Fakültesi, İş Sağlı̆ı ve Güvenliği Bölümü, İstanbul, Türkiye, (ORCID: 0000-0002-5633-2296), \\ beyrul.canbaz@yeniyuzyil.edu.tr
}

(İlk Geliş Tarihi 24 Aralık 2021 ve Kabul Tarihi 25 Ocak 2022)

(DOI: $10.31590 /$ ejosat.1043746)

\begin{abstract}
ATIF/REFERENCE: Canbaz, B., (2022). Genel Hizalama İndeksi Yöntemiyle 2 Boyutlu Saf Fermiyonik Modelde Kaosun
\end{abstract} İncelenmesi. Avrupa Bilim ve Teknoloji Dergisi, (33), 161-166.

$\ddot{O} \mathbf{z}$

Thirring model 2 boyutlu konformal invaryant saf fermiyonik bir modeldir. Bu modelde Heisenberg yaklaşımı kullanılarak spinör tipi instantonlara karşılık gelen çözümler elde edilmiştir. İnstantonlar standart modelde kuantum alan teorisi bağlamında var olan klasik topolojik çözümlerdir. İnstantonlar farklı vakumlar arasındaki tünellemeye karşılık geldikleri için kuarkların parçacıklar içinde hapsolmasını açıklamada önemli bir rol oynar. Bu çalışmada Thirring modelden yararlanarak elde edilen spinör tipi instanton çözümlerinin dinamiği hakkında daha fazla bilgi elde etmek amaçlanmaktadır. Spinör tipi instanton çözümleri lineer olmayan çözümlerdir. Düzenli ve kaotik hareketi hızlı bir şekilde ayırt edebilmesi ve yarı periyodik hareketin meydana geldiği torusun boyutsallığını belirlemesi Genelleştirilmiş Hizalama İndeksi (Generalized Alignment Index) yöntemini diğer yöntemlere göre öne çıkarmaktadır. Bu çalışmada GALI yöntemi kullanılarak spinör tipi instanton çözümlerinin kaotik davranışı karakterize edilmektedir.

Anahtar Kelimeler: İnstanton, Spinör, Kaos, Lineer Olmayan Sistemler, Genelleştirilmiş Hizalama İndeksi.

\section{Investigation Chaos by General Alignment Index Method in 2D Pure Fermionic Model}

\begin{abstract}
The Thirring model is a 2D conformal invariant pure fermionic model. In this model, solutions corresponding to spinor type instantons are obtained by using Heisenberg ansatz. Instantons are classical topological solutions that exist in the context of quantum field theory in the standard model. Since instantons correspond to tunneling between different vacuums, they play an important role in explaining the entrapment of quarks within particles. In this study, it is aimed to get more information about the dynamics of spinor type instanton solutions obtained by using the Thirring model. Spinor-type instanton solutions are non-linear solutions. The ability to quickly distinguish between regular and chaotic motion and determine the dimensionality of the torus in which quasi-periodic motion occurs makes the Generalized Alignment Index method stand out compared to other methods. In this study, the chaotic behavior of spinor-type instanton solutions is characterized using the GALI method.
\end{abstract}

Keywords: Instanton, Spinor, Chaos, Nonlinear Dynamics, Generalized Alignment İndex.

\footnotetext{
*Sorumlu Yazar: beyrul.canbaz@yeniyuzyil.edu.tr
} 


\section{Giriş}

Solitonlar instantonlara yakından ilgili olan, sonlu enerjili ve kuantum durumları önemli topolojili klasik konfigürasyonlardır. İnstantonlar ve solitonlar, topolojik yükler olarak bilinen bir dizi topolojik değişmez tarafindan sinıflandırılır (Fradkin, 2021). Bu topolojik değişmezler genellikle kuantum sapmalarının bir sonucu olarak teorinin eylemine dahil edildiklerinde, teorinin davranışını derinden değiştirirler.

İnstantonların klasik olarak topolojik solitonlar olduğu bilinmektedir (Fradkin, 2021). İnstantonlar hem küresel simetri teorilerinde hem de ayar teorilerinde ve faz geçişleri teorisinde yol integralinin pertürbatif olmayan tanımında kilit rol oynayan topolojik uyarıları tanımlar (Dunajski, 2010). İnstantonlar, sıfır enerji ile sonlu bir etkiye sahip olduklarından, uzay-zamanda farklı topolojilere sahip boşluklar arasında tünel etkisi sağlayan kuantum alanlarının konfigürasyonları olarak da kabul edilmiştir (Belavin ve ark., 1975). Özellikle, instantonların bu özelliği, kuarkların hapsolma problemini yorumlar.

Fiziğin çeşitli alanlarında dinamik sistemler birçok değişik uygulama ile oldukça önemli ve geniş bir araştırma alanı oluşturmaktadır. Birçok dinamik sistemde hareketin düzenli veya kaotik doğasının hızlı ve verimli tespiti yıllar boyunca çok aktif bir araştırma konusu olmuştur. Zaman içinde genişleyen ve gelişen dinamik sistemlerin bir alt alanı olarak kaos algılama yöntemleri ele alınmıştır. $\mathrm{Bu}$ çalışmada, kaos tespiti için kullanılan Genelleştirilmiş Hizalama İndeksi (Generalized Alignment Index) (GALI) ile çalışılmıştır. Bu yöntem hızlı ve verimli sonuçlar vermesinden dolayı tercih edilmiştir. GALI, kaotik ve düzenli hareketler arasındaki fark, zayıf kaotik davranışı erken tespit edebilmesi, meydana gelen torusun boyutsallığını belirleyebilmesi gibi özellikler sunar.

GALI, belirli bir yörüngeden birkaç sapma vektörünün evrimini gerektirir. Kullanılan vektörlerin $\mathrm{k}$ sayısı, indeksin sırasını, yani $G A L I_{k}{ }^{\prime} 1$ tanımlar. Düzenli hareket durumunda $G A L I_{k}, \mathrm{k}$ için hareketin gerçekleştiği torusun teğet uzayının $\mathrm{N}$ boyutundan büyük olmayan, sonunda pozitif bir değer etrafinda salınım yaparken, kaotik yörüngeler için katlanarak hızlı bir şekilde sıfıra yönelir (Skokos ve ark., 2007). Kararlı periyodik yörüngeleri durumunda, $G A L I_{k}$ belirli güç yasalarını izleyerek sıfır olma eğilimindeyken, kararsız periyodik yörüngeler için katlanarak hızlı bir şekilde sıfır olur (Skokos ve Bountis, 2008).

\section{Materyal ve Metot}

\subsection{Thirring Model}

Thirring model, iki boyutlu fermiyon-fermiyon etkileşimleri, konformal invaryant Lagrange

$$
L=i \bar{\psi} \sigma_{\mu} \partial_{\mu} \psi+\frac{g}{2}(\bar{\psi} \psi)^{2}
$$

denklemi ile tanımlanır (Thirring, 1958). Burada g pozitif kuplaj sabitidir ve fermiyon alanı $\psi(x) 1 / 2$ ölçek boyutuna sahiptir (Akdeniz ve Smailagic, 1979). Hareket denklemi ise,

$$
i \sigma_{\mu} \partial_{\mu} \psi+g(\bar{\psi} \psi) \psi=0
$$

ile verilir. Heisenberg yaklaşımının (Canbaz ve ark., 2012) Öklid konfigürasyonunun kompleks biçimi,

$$
\psi=\left[i x_{\mu} \gamma_{\mu} \chi(s)+\varphi(s)\right] C
$$

denklemi ile verilir. Burada $C$ keyfi seçilmiş bir spinör sabiti, $\chi(s)$ ve $\varphi(s)$ ise $s=x^{2}+t^{2}\left(x_{1} \equiv x, x_{2} \equiv t\right)$ 'nin gerçek fonksiyonlarıdır. (3) denklemini (2) denkleminde yerine koyarsak,

$$
\chi(s)+s \frac{d \chi(s)}{d s}+\alpha\left[s \chi(s)^{2}+\varphi(s)^{2}\right] \varphi(s)=0
$$

$\frac{d \varphi(s)}{d s}-\alpha\left[s \chi(s)^{2}+\varphi(s)^{2}\right] \chi(s)=0$

elde ederiz. Burada $\alpha=g(\bar{C} C)$ yazarsak,

$$
\begin{aligned}
& i ð \psi=i \sigma_{\mu} \partial_{\mu} \psi=\left[-2 \chi(s)-2 s \frac{d \chi(s)}{d s}+2 i x_{\mu} \sigma_{\mu} \frac{d \varphi(s)}{d s}\right] \bar{C} C \\
& (\bar{\psi} \psi)=\left(s \chi(s)^{2}+\varphi(s)^{2}\right)(\bar{C} C)
\end{aligned}
$$

buluruz. $\chi=A s^{-\sigma} p(t)$ ve $\varphi=B s^{-\tau} q(t)$ ile $t \equiv \ln s$ ve $\sigma=$ $\tau+1 / 2, \tau=1 / 4$ (Canbaz ve ark., 2012) yazarak, doğrusal olmayan basit diferansiyel denklem sistem çifti (4a) ve (4b) boyutsuz formunu,

$$
\begin{aligned}
& 2 \frac{d p(t)}{d t}+\frac{1}{2} p(t)-\alpha A B\left(p(t)^{2}+q(t)^{2}\right) q(t)=0, \\
& 2 \frac{d q(t)}{d t}-\frac{1}{2} q(t)-\alpha A B\left(p(t)^{2}+q(t)^{2}\right) p(t)=0
\end{aligned}
$$

elde ettik. Burada p ve q, t'nin boyutsuz fonksiyonlarıdır ve A, B sabitlerdir (Canbaz ve ark., 2012). (6a) ve (6b)'deki denklem sistemine, Thirring doğrusal olmayan diferansiyel denklem sistemi diyoruz. $\beta=\alpha(\mathrm{AB})=1$ için bu denklem sisteminin çözümü, (Canbaz ve ark., 2012) de verilen Thirring instantonlarıdır. Son zamanlarda, faz uzayında Thirring instantonlarının evriminde kuplaj sabitinin rolü araştırılmıştır (Canbaz ve ark., 2012).

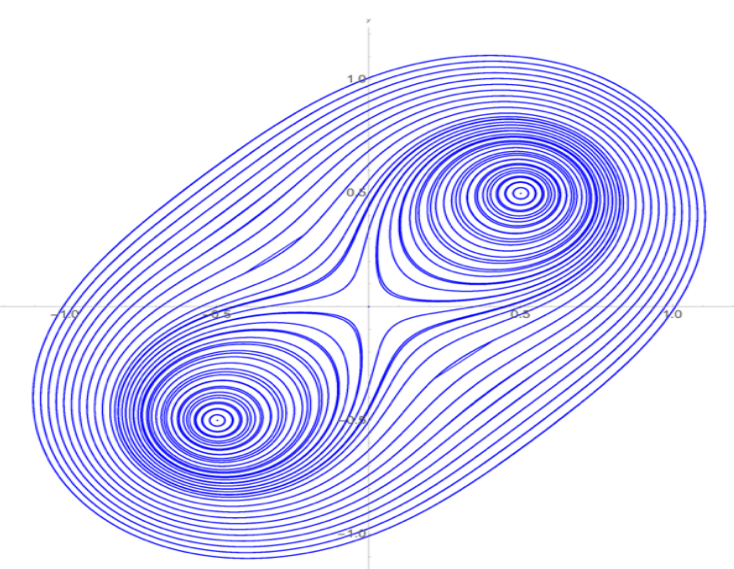

Şekil 1. Thirring instantonlarının çözümlerine karşılık gelen faz diyagramı (Canbaz ve ark., 2012) (Figure 1. Phase diagram corresponding to the solutions of Thirring instantons (Canbaz et al., 2012).) 


\subsection{Genelleştirilmiş Hizalama (Generalized Alignment Index) (GALI)}

GALI yönteminde vektör uzayının $2 \mathrm{~N}$ boyutlu uzayında $\mathrm{S}$ bir yörüngenin değişimi fark denklemi tarafindan yönetilir.

$\vec{x}(n+1) \equiv \vec{x}_{n+1}=F\left(\vec{x}_{n}\right)$

Bu durumda, bir $\vec{x}_{n}$ referans yörüngesine göre bir sapma vektörü $\vec{\omega}(n) \equiv \vec{\omega}_{n}$ 'nin değişimi, karş1lık gelen teğet vektör uzayı tarafindan verilir.

$\vec{\omega}(n+1) \equiv \vec{\omega}_{n+1}=\frac{\partial F}{\partial \vec{x}}\left(\vec{x}_{n}\right) \cdot \vec{\omega}_{n}$

$\mathrm{N}$ serbestlik derecesi Hamiltonian akışları ve $2 \mathrm{~N}$ boyutlu vektör uzayı için $\mathrm{k}\left(G A L I_{k}\right)$ düzeyindeki Genelleştirilmiş Hizalama İndeksi, $2 \leq \mathrm{k} \leq 2 \mathrm{~N}$, başlangıçta doğrusal olarak bağımsız $\mathrm{k}$ sapma vektörlerinin $\vec{\omega}_{k}(0)$ değişimi yoluyla belirlenir. Taşma problemlerinden kaçınmak için, ortaya çıkan sapma vektörleri $\vec{\omega}_{k}(t)$ sürekli olarak normalize edilir, ancak yönleri bozulmadan tutulur. Daha sonra, (Skokos ve ark., 2007) 'a göre $G A L I_{k}$, kenarları $\mathrm{k}$ birim sapma vektörleri $\widehat{\omega}_{i}(t)=$ $\widehat{\omega}_{i}(t) /\left\|\widehat{\omega}_{i}(t)\right\|, \quad i=1,2, \ldots, k$ olan k-paralelkenarının hacmi olarak tanımlanır ve bu vektörlerin vektörel çarpımı ile şu şekilde belirlenir:

$$
\operatorname{GALI}_{k}(t)=\left\|\widehat{\omega}_{1}(t) \wedge \widehat{\omega}_{2}(t) \wedge \ldots \wedge \widehat{\omega}_{k}(t)\right\|
$$

ve $\|$.$\| olağan klasik normu gösterir. \mathrm{Bu}$ tanımdan, sapma vektörlerinden en az ikisinin lineer bağımlı hale gelmesi durumunda, denklem (11)'deki vektörel çarpımının sıfır olduğu ve $G A L I_{k}{ }^{\prime}$ 'in ortadan kalktığı açıktır.

Bir N serbestlik derecesi Hamiltonian akışının veya bir $2 \mathrm{~N}$ boyutlu vektör uzayı $2 \mathrm{~N}$ boyutlu faz uzayında $\mathrm{S}$, düzenli yörüngeler, Hamilton akışları için $2 \leq \mathrm{s} \leq \mathrm{N}$ üzerinde bulunur. $\mathrm{Bu}$ tür yörüngeler için, tüm sapma vektörleri, hareketin üzerinde bulunduğu torusun s boyutlu teğet uzayına düşme eğilimindedir. Böylece, $\mathrm{k} \leq \mathrm{s}$ genel sapma vektörleri ile başlarsak, lineer bağımlı hale gelmeleri için özel bir neden olmadığından bunlar torusun s-boyutlu teğet uzayında lineer olarak bağımsız kalacaktır. Sonuç olarak $G A L I_{k}$ pratik olarak sabit kalır ve $\mathrm{k} \leq \mathrm{s}$ için sıfırdan farklıdır. Öte yandan, bazı sapma vektörlerinin eninde sonunda lineer bağımlı hale gelmesi gerekeceğinden, $G A L I_{k} \mathrm{k}>\mathrm{s}$ için sıfır olma eğilimindedir. Özellikle, s-boyutlu teğet uzayı üzerinde uzanan yarı düzenli yörüngeler için $G A L I_{k}$ 'in genel davranışı (Skokos ve Manos, 2016), (Christodoulidi ve Bountis, 2006) ile verilmektedir.

$$
G A L I_{k}(t) \propto\left\{\begin{array}{cc}
\text { sabit } & 2 \leq k \leq s \\
\frac{1}{t^{k-s}} & s<k \leq 2 N-s \\
\frac{1}{t^{2(k-N)}} & 2 N-s<k \leq 2 N
\end{array}\right.
$$

$\mathrm{Bu}$ tahminlerin yalnızca yukarıda belirtilen koşullar tam olarak karşılandığında geçerli olduğunu unutulmamalıdır. Örneğin, mümkün olan tek torusun bir $\mathrm{s}=1$ boyutlu invaryant eğri olduğu 2 boyutlu vektör uzayı durumunda, teğet uzayı 1 boyutludur. $\mathrm{Bu}$ nedenle, $G A L I_{2}$ 'nin davranışı bu durumda tek olası indekstir, denklem (12)'nin üçüncü dalı, yani $G A L I_{2} \propto$ $1 / t^{2}$ tarafindan verilir, çünkü denklem (12)'nin ilk iki durumu uygulanabilir değildir. Denklem (12)'den, N-boyutlu bir torus üzerinde uzanan normal yörüngelerin düzenli durumu için, $G A L I_{k}$ 'in davranışının şu şekilde verildiğini çıkarıyoruz: (Moges, 2020)

$G A L I_{k}(t) \propto\left\{\begin{array}{lc}\text { sabit } & 2 \leq k \leq N \\ \frac{1}{t^{2(k-N)}} & N<k \leq 2 N\end{array}\right.$

$G A L I_{k}{ }^{\prime}$ 'in düşük boyutlu tori, yani $\mathrm{k}<\mathrm{N}$ ile $\mathrm{k}$ boyut torus üzerindeki düzenli yörüngeler için davranışının (Manos ve ark., 2012) tarafından verildiğini göz önünde bulundurulmaktadır.

$G A L I_{k}(t) \propto\left\{\begin{array}{cc}\frac{1}{t^{(k-1)}} & 2 \leq k \leq 2 N-1 \\ \frac{1}{t^{2 N}} & k=2 N\end{array}\right.$

Öte yandan, kaotik bir yörünge için tüm sapma vektörleri, kendilerini maksimum Lyapunov karakteristik üssü tarafından tanımlanan yönde hizalayarak lineer olarak bağımlı hale gelme eğilimindedir ve bu nedenle, bu durumda, $G A L I_{k}$ yasayı izleyerek üssel olarak sıfır olma eğilimindedir (Manos ve ark., 2012)).

$G A L I_{k}(t) \propto e^{-\left[\left(\sigma_{1}-\sigma_{2}\right)+\left(\sigma_{1}-\sigma_{3}\right)+\cdots+\left(\sigma_{1}-\sigma_{k}\right)\right] t}$

Burada $\sigma_{1}, \ldots, \sigma_{k}$ yörüngenin ilk en büyük $\mathrm{k}$ Lyapunov karakteristik üsleridir. Özellikle $\mathrm{k}=2$ olduğunda, $G A L I_{2}$ aşağıdaki gibi katlanarak hızlı bir şekilde sıfırlanma eğilimindedir:

$G A L I_{2}(t) \propto e^{-\left(\sigma_{1}-\sigma_{2}\right) t}$

$G A L I_{2}$ 'nin 2 boyutlu eşleştirmelerde düzenli yörüngeler için davranışı özel dikkat gerektirir. Bu durumda hareket 1 boyutlu torus üzerinde mevcut olduğundan, iki sapma vektörü yine 1 boyutlu olan bu torusun teğet uzayına düşme eğilimindedir. Böylece iki vektör sonunda kaotik yörüngelerde olduğu gibi lineer olarak bağımlı hale gelecektir, ancak bu (Skokos ve Manos, 2016) tarafından verilen farklı bir zaman oranı ile olur.

$G A L I_{2}(n) \propto \frac{1}{n^{2}}$

Burada n yineleme sayısıdır. $G A L I_{k}$ değerini hesaplamanın etkili bir yolu, sütunlar olarak k birim vektör

$\widehat{\omega}_{i}(t)=\frac{\omega_{i}(t)}{\left\|\omega_{i}(t)\right\|}=\left(\widehat{\omega}_{i, 1}, \widehat{\omega}_{i, 2}, \ldots, \widehat{\omega}_{i, 2 N}\right)$ 
$A^{T}=U Z V^{T}$

koordinatlarını içeren matrisin Tekil Değer Ayrışımı (TDA) yolu ile gerçekleşir (Christodoulidi ve Bountis, 2006)

$A=\left(\begin{array}{llll}\widehat{\omega}_{1} & \widehat{\omega}_{2} & \ldots & \widehat{\omega}_{k}\end{array}\right)=\left(\begin{array}{cccc}\widehat{\omega}_{1,1} & \widehat{\omega}_{2,1} & \ldots & \widehat{\omega}_{k, 1} \\ \widehat{\omega}_{1,2} & \widehat{\omega}_{2,2} & \ldots & \widehat{\omega}_{k, 2} \\ \vdots & \vdots & & \vdots \\ \widehat{\omega}_{1,2 N} & \widehat{\omega}_{2,2 N} & \ldots & \widehat{\omega}_{k, 2 N}\end{array}\right)$

Bunun sonucunda, $G A L I_{k}$ değerinin şu şekilde verilebileceği görülmektedir:

$\operatorname{GALI}_{k}(t)=\sqrt{\operatorname{det}\left[A(t) \cdot A^{T}(t)\right]}$

Burada ' $\operatorname{det}(A)$ ' bir A matrisinin determinantını gösterir. Denklem 19'daki matris çarpımı bir $\mathrm{k} \times \mathrm{k}$ simetrik pozitif tanımlı matristir.

$A A^{T}=\left[\begin{array}{ccccc}\left\langle\widehat{\omega}_{1}, \widehat{\omega}_{1}\right\rangle & \left\langle\widehat{\omega}_{1}, \widehat{\omega}_{2}\right\rangle & \left\langle\widehat{\omega}_{1}, \widehat{\omega}_{3}\right\rangle & \ldots & \left\langle\widehat{\omega}_{1}, \widehat{\omega}_{k}\right\rangle \\ \left\langle\widehat{\omega}_{1}, \widehat{\omega}_{2}\right\rangle & \left\langle\widehat{\omega}_{2}, \widehat{\omega}_{2}\right\rangle & \left\langle\widehat{\omega}_{2}, \widehat{\omega}_{3}\right\rangle & \ldots & \left\langle\widehat{\omega}_{2}, \widehat{\omega}_{k}\right\rangle \\ \vdots & \vdots & \vdots & \ldots & \vdots \\ \left\langle\widehat{\omega}_{1}, \widehat{\omega}_{k}\right\rangle & \left\langle\widehat{\omega}_{2}, \widehat{\omega}_{k}\right\rangle & \left\langle\widehat{\omega}_{3}, \widehat{\omega}_{k}\right\rangle & \ldots & \left\langle\widehat{\omega}_{k}, \widehat{\omega}_{k}\right\rangle\end{array}\right]$

i. satır ve j. sütunun her bir elemanı, $\widehat{\omega}_{i}$ ve $\widehat{\omega}_{j}$ birim sapma vektörlerinin iç çarpımıdır, böylece

$\left\langle\widehat{\omega}_{i}, \widehat{\omega}_{j}\right\rangle=\cos \theta_{i j} \quad, \quad i, j=1,2, \ldots, k$

ile verilir. Burada $\theta_{i j}, \widehat{\omega}_{i}$ ve $\widehat{\omega}_{j}$ vektörleri arasındaki açıdır. Böylece denklem (20)'nin matrisi şu şekilde yazılabilir:

$A A^{T}=\left[\begin{array}{ccccc}1 & \cos \theta_{12} & \cos \theta_{13} & \ldots & \cos \theta_{1 k} \\ \cos \theta_{12} & 1 & \cos \theta_{23} & \ldots & \cos \theta_{2 k} \\ \vdots & \vdots & \vdots & \ldots & \vdots \\ \cos \theta_{1 k} & \cos \theta_{2 k} & \cos \theta_{3 k} & \ldots & 1\end{array}\right]$

$G_{A L I}$ değeri, Denklem (11)'de tanımlanan k sapma vektörlerinin vektörel çarpımının normu ile hesaplanabilir.

$\operatorname{GALI}_{k}=\left\{\sum_{1 \leq i_{1}<i_{2}<\cdots i_{k} \leq 2 N}\left(\operatorname{det}\left[\left(\begin{array}{cccc}\omega_{1_{i 1}} & \omega_{1_{i 2}} & \ldots & \omega_{1_{i k}} \\ \omega_{2_{i 1}} & \omega_{2_{i 2}} & \ldots & \omega_{2_{i k}} \\ \vdots & \vdots & & \vdots \\ \omega_{k_{i 1}} & \omega_{k_{i 2}} & \ldots & \omega_{k_{i k}}\end{array}\right)\right]\right)^{2}\right\}^{1 / 2}$

Burada toplam, $2 \mathrm{~N}$ dişındaki tüm olası $\mathrm{k}$ indeks kombinasyonları üzerinden yapılır. $\mathrm{Bu}$, hesaplamamızda A'nın tüm olası $\mathrm{k} \times \mathrm{k}$ determinantlarını göz önünde bulundurmamız gerektiği anlamına gelir. Pratik bir bakış açısından bu teknik, denklem (23)' deki çok sayıda determinanttan dolayı birçok serbestlik dereceli sistemler için sayısal olarak verimli değildir. Yine de Denklem (23), (Skokos ve ark., 2007)'da gösterildiği gibi, GALI'nin kaotik ve düzenli yörüngeler için asimptotik davranışının teorik yaklaşımı için idealdir.

Tekil Değer Ayrışımı (TDA) yöntemine (Skokos, ve ark. 2007) göre $2 \mathrm{~N} \times \mathrm{k}$ matrisi $A^{T}, 2 \mathrm{~N} \times \mathrm{k}$ sütun-ortogonal matris $\mathrm{U}$, $\mathrm{k} \times \mathrm{k}$ diyagonal matris Z'nin negatif olmayan gerçek sayılarla $Z_{i}$, $i=1,2, \ldots, k$ çarpımı olarak köşegeni üzerinde yazılabilir ve $\mathrm{k} \times$ $\mathrm{k}$ ortogonal matris V'nin transpozesi:

e-ISSN: 2148-2683
Daha sonra $G A L I_{k}$, denklem (19) ve (24) kullanılarak U ve V ortogonal olduğundan, $U^{T} \cdot U=V^{T} . V=I_{k}, I_{k}$ 'nin

$\mathrm{k} \times \mathrm{k}$ birim matrisi olduğu akılda tutularak hesaplanır. Böylece,

$G A L I_{k}=\sqrt{\operatorname{det}\left(A A^{T}\right)}$,

$=\sqrt{\operatorname{det}\left[\left(V Z^{T} U^{T}\right) \cdot\left(U Z V^{T}\right)\right]}$,

$=\sqrt{\operatorname{det}\left[\operatorname{Vdiag}\left(z_{i}^{2}\right) V^{T}\right]}$,

$=\sqrt{\operatorname{det}\left[\operatorname{diag}\left(z_{i}^{2}\right)\right]}$,

$=\prod_{i=1}^{k} z_{i}$

Burada $z_{i}, i=1, \ldots, k$, TDA prosedürü ile elde edilen A'nın sözde tekil değerleridir.

\section{Araştırma Sonuçları ve Tartışma}

Thirring modele Heisenberg yaklaşımı uygulayarak bulunan spinör tipi instanton çözümleri baz alınarak GALI yöntemi ile yörüngelerin durumu araştırıldı. Şekil 1'deki faz diyagramında görüldüğü gibi denge noktaları $(0.5,0.5),(-0.5,-0.5)$ tir. Kararlı noktalara çok yakın ve biraz daha uzaktaki yörüngelerin durumlarına bakıldı.

a)
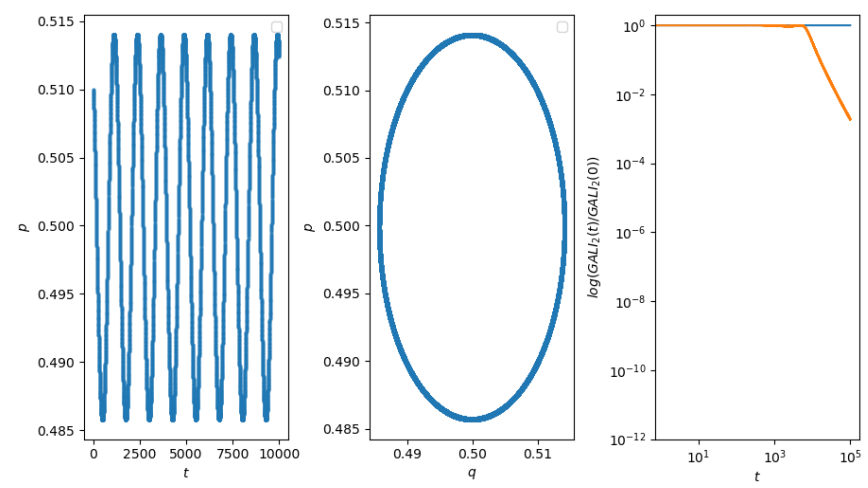

b)
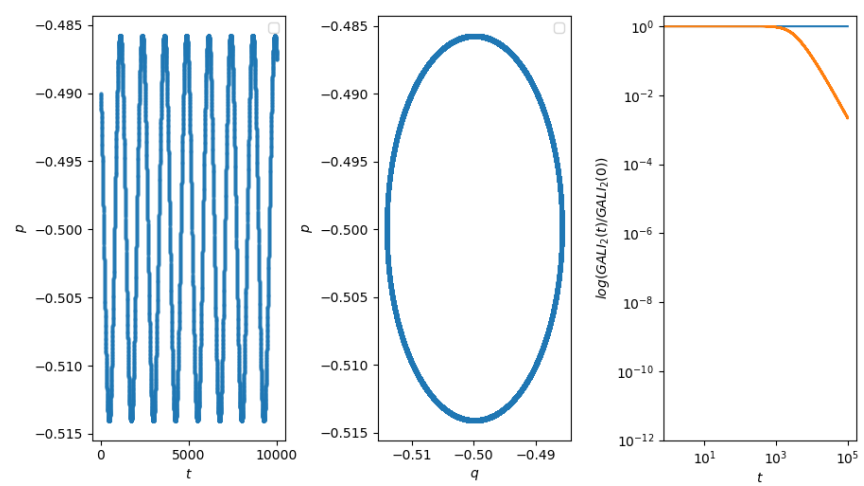

Şekil 2. $\beta=1$ sabit değeri; a) başlangıç koşulları (0.51, 0.49); strasiyla Zaman Serisi, Faz Diyagrami, $10^{5}$ iterasyon sayısina göre GALI ${ }_{2}$ nin evrimi, b) başlangıç koşulları (-0.49, -0.51); strasiyla Zaman Serisi, Faz Diyagramı, $10^{5}$ iterasyon sayısına göre $\mathrm{GALI}_{2}$ nin evrimi 
(Figure 2. The constant value of $\beta=1$; a) initial conditions $(0.51$, 0.49); respectively, Time Series, Phase Diagram, evolution of $G \mathrm{ALI}_{2}$ according to the number of $10^{5}$ iterations, $\left.b\right)$ initial conditions (-0.49, -0.51); respectively, Time Series, Phase Diagram, evolution of $G A L I_{2}$ according to the number of $10^{5}$ iterations.)

Şekil 2 de görüldüğü gibi a ve b denge durumları $(0.5,0.5)$, ($0.5,-0.5$ ) (Canbaz ve ark., 2012) civarında periyodik yörüngelere sahip olduğu gözlenmektedir. Her iki kararlı noktalara yakın durumlarda zaman serisi ve faz diyagramında periyodik olarak hareket ettiği görülmektedir. GALI indisi uzun süre sabit kalmaktadır, sonrasında azalma eylemi göstermektedir. $\mathrm{Bu}$ durum denge noktaları etrafinda periyodik bir yörüngeye sahip olduğunu sonucunu ortaya çıkarmaktadır.

a)
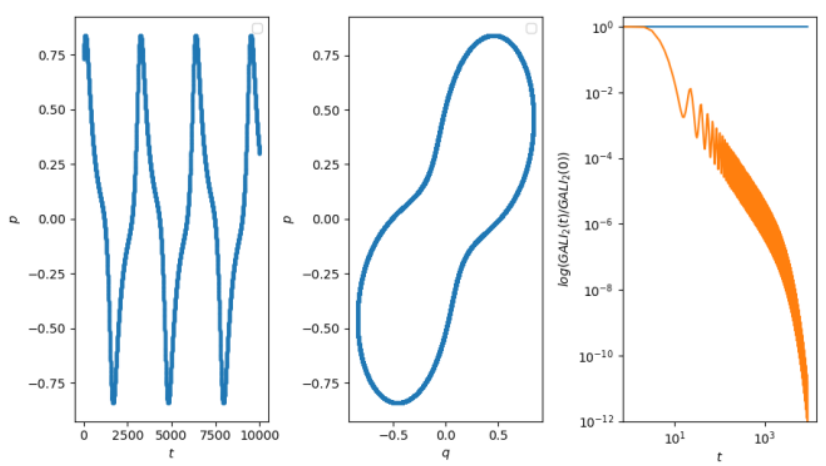

b)
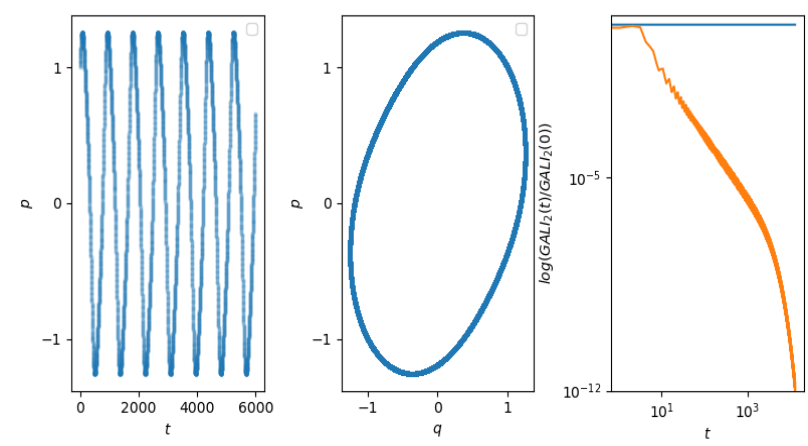

c)
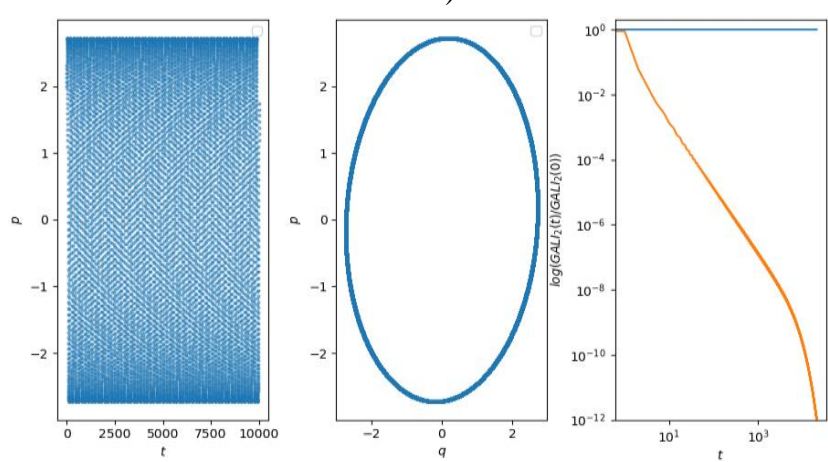

Şekil 3. $\beta=1$ sabit değeri; a) başlangıç koşulları (0.73, 0.73); sirasiyla Zaman Serisi, Faz Diyagramı, $10^{5}$ iterasyon sayısina göre GALI ${ }_{2}$ nin evrimi, b) başlangıç koşulları (1.0, 1.0); sirasiyla Zaman Serisi, Faz Diyagramı, $10^{5}$ iterasyon sayısına göre $\mathrm{GALI}_{2}$ nin evrimi, c) başlangıç koşulları (1.99, 1.99); e-ISSN: 2148-2683 strasiyla Zaman Serisi, Faz Diyagramı, $10^{5}$ iterasyon sayısina göre $\mathrm{GALI}_{2}$ nin evrimi. (Figure 3. The constant value of $\beta=1$; a) initial conditions $(0.73,0.73)$; respectively, Time Series, Phase Diagram, evolution of $\mathrm{GALI}_{2}$ according to $10^{5}$ iteration number, b) initial conditions (1.0, 1.0); Time Series, Phase Diagram, evolution of $\mathrm{GALI}_{2}$ according to $10^{5}$ iteration number, respectively, c) initial conditions $(1.99,1.99)$; respectively, Time Series, Phase Diagram, evolution of $\mathrm{GALI}_{2}$ according to the number of $10^{5}$ iterations.)

Şekil 3 'te görüldüğü gibi a, b ve c denge durumlarından $(0.5,0.5),(-0.5,-0.5)$ (Canbaz ve ark., 2012) uzaklaştıkça yörüngeler kaotiktir. Zaman serisi ve faz diyagramları net olarak periyodiklik veya kaotiklik hakkında bahsetmek mümkün değildir. a) grafiğindeki GALI indisi bir süre sonra düşmeye başlamıştır. b) grafiğindeki GALI indisi de aynı şekilde bir süre sonra düşmeye başlamıştır. c) grafiğindeki GALI de daha kısa bir süre sonra düşme eylemi başlamıştır. Bu durumlar kaotikliğin göstergesidir (Skokos ve Bountis, 2008). Şekil 3'te denge durumundan farklı 3 başlangıç değerine göre incelendiğinde; denge noktalarından uzaklaştıkça yörüngelerin kaotikleştiği görülmektedir.

\section{Sonuç}

İnstantonlar uzay-zamanda farklı topolojik vakumlar arasındaki tünellemeyi sağlayan kuantum alan teorisinde parçacık benzeri çözümlerdir. Kuantum ayar teorisinde vakumlar arası tünelleme önemlidir (Shifman, 1994). Bu çalışmada Thirring modele Heisenberg yaklaşımı uygulayarak bulunan spinör tipi instanton çözümlerinin dinamiğini daha iyi anlamak amaçlanmıştır. $\mathrm{Bu}$ dinamiği daha iyi anlamak için Genelleştirilmiş hizalama indeksi yöntemi kullanılmıştır. Genelleştirilmiş hizalama indeksi yöntemi yüksek boyutlu sistemlerde olduğu gibi düşük boyutlu sistemlerde de düzenli ve kaotik hareketi araştırmada uygun bir yöntem olarak seçilmiştir.

Daha önceki yapılan çalışmada faz uzayına bakıldı (Canbaz ve ark., 2012). Faz uzayı çekicileri gösterebilmektedir ancak yörüngeler hakkında detaylı bilgi vermemektedir. Zaman serisi ve faz diyagramı yörüngelerin düzenli veya kaotikliği hakkında kesin bir bilgi vermediğinden GALI yönteminden yararlanıldı. GALI yöntemi sayesinde yörüngelerin dinamiği hakkında daha detaylı bilgi verdiğinden bu çalışmada spinör tipi instanton çözümleri yörüngeleri hakkında daha fazla bilgiye sahip olduk. Denge durumu $(0.5,0.5),(-0.5,-0.5)$ civarında düzenli olmasına karşın denge durumundan uzaklaştıkça yörüngeler kaotik olmaktadir.

İki boyutlu konformal invaryant saf fermiyonik spinör tipi instantonların başlangıç koşullarına hassas bağlılığ görülmektedir. Hem düzenli hem de kaotik olması dinamiğinin evriminin kaotik olduğu sonucunu göstermektedir. Bu çalışma kuantum alan teorilerinde vakum durumundaki çözümlerin kaotik özelliklere sahip olduklarına dair bir göstergedir.

\section{Teșekkür}

$\mathrm{Bu}$ taslağı hazırlarken verdiği destek için K. Gediz Akdeniz'e teşekkür ederim. 


\section{Kaynakça}

Akdeniz, K. G., Smailagic, A., (1979). Classical solutions for fermionic models. Il Nuovo Cimento A, 51(3), 345- 357

Belavin, A.A., Polyakof, A.M., Schwartz, Schwartz, A.S., Tyupkin Yu.S., (1975). Pseudoparticle solutions of YangMills equations. Phys. Lett., B, 59(1), 85-87

Canbaz, B., Onem, C., Aydogmus, F., Akdeniz, K. G., (2012). From Heisenberg ansatz to attractor of Thirring Instanton. Chaos, Solitons \& Fractals, 45(2), 188-191

Christodoulidi, H., Bountis, T., (2006). Low-dimensional quasiperiodic motion in Hamiltonian systems. ROMAI Journal 2(2), 37-44.

Dunajski, M., (2010). Solitons, Instantons, and Twistors. Oxford University Press, New York

Fradkin, E., (2021). Quantum Field Theory: An Integrated Approach. Princeton University Press

Manos, T., Skokos, C., Antonopoulos, C., (2012). Probing the local dynamics of periodic orbits by the generalized alignment index (GALI) method. International Journal of Bifurcation and Chaos, 22(9), 1250218

Moges, H.T., (2020). Investigating Chaos by the Generalized Alignment Index (GALI) Method

Shifman, M., (1994). Instantons in Gauge Theories. World Scientific Publishing Company

Skokos, C., Bountis, T, Antonopoulos, C., (2007). Geometrical properties of local dynamics in Hamil- tonian systems: The Generalized Alignment Index (GALI) method. Physica D, 231(1), 30-54

Skokos, C., Bountis, T., Antonopoulos, C., (2008). Detecting chaos, determining the dimensions of tori and predicting slow diffusion in Fermi-Pasta-Ulam lattices by the Generalized Alignment Index method. The European Physical Journal Special Topics, 165(1), 5

Skokos, C., Manos, T., (2016). The Smaller (SALI) and the Generalized (GALI) alignment indices: Efficient methods of chaos detection. Lect. Notes Phys. 915, 129

Thirring W. E., (1958). A Soluble. Relativistic Field Theory Anal. Phys., 3, 91. 